ISSN: 1130-3743 - e-ISSN: 2386-5660

DOI: http://dx.doi.org/10.14201/teoredu292127144

\title{
COMPONENTES SOCIOLINGÜÍSTICOS Y CULTURALES EN EL PROCESO EDUCATIVO DEL ALUMNADO INMIGRANTE ${ }^{1}$
}

\section{Sociolinguistic and cultural components in the educative process of immigrant students}

\section{Composantes sociolinguistiques et culturelles dans le processus éducatif des élèves immigrants}

\author{
Elena MARTín-PASTOR*, Cristina JENARO RÍO** y Francisca GONZÁLEZ-GIL* \\ * Universidad de Salamanca. Facultad de Educación. Departamento de \\ Didáctica, Organización y Métodos de Investigación. C/ Paseo de Canalejas, 169. \\ 37008 Salamanca.emapa@usal.es,frang@usal.es \\ ** Universidad de Salamanca. Facultad de Psicología. Departamento de \\ Personalidad, Evaluación y Tratamientos Psicológicos. Avda. Merced, 109-131. \\ 37005 Salamanca.crisje@usal.es
}

Fecha de recepción: enero de 2017

Fecha de aceptación: marzo de 2017

\section{RESUMEN}

El alumnado inmigrante en el sistema educativo español es uno de los protagonistas de los altos índices de fracaso escolar. Ante esta situación, partimos del supuesto de que parte de las dificultades que pueden presentar estos alumnos se justifiquen por su desconocimiento de los códigos culturales y los mediadores comunicacionales que determinan el funcionamiento de las escuelas, los cuales dificultan su acceso a los contenidos didácticos, su comunicación, participación e

1. Investigación financiada por la Junta de Castilla y León y el Fondo Social Europeo. 
inclusión socioeducativa. Nuestro trabajo tiene por objetivo identificar semejanzas y diferencias en la comprensión de componentes sociolingüísticos y socioculturales de niños procedentes de países de habla hispana, frente a sus compañeros autóctonos de Educación Secundaria Obligatoria. Para ello se contó con la participación de 247 alumnos de Salamanca, de los cuales 141 eran inmigrantes. Los resultados revelan que los estudiantes extranjeros hispanoparlantes presentan mayores dificultades para inferir el significado de vocabulario y expresiones que se manejan en los recursos didácticos. Lo anterior nos lleva a replantearnos las prácticas educativas que se están desarrollando para responder a las necesidades de estos escolares.

Palabras clave: alumnado inmigrante; diversidad sociolingüística y cultural; educación intercultural; fracaso escolar.

\section{SUMMARY}

In the Spanish educational system, immigrant students are one of the principal groups with high rates of school failure. In this situation we assume that part of the difficulties of these students can be explained by their ignorance of cultural codes and the communication mediators in which the school is organized which can impede their access to the curricula, their communication, their participation and their social and educative inclusion. Our study aims to identify similarities and differences in the understanding of sociolinguistic and sociocultural components among immigrant students whose mother tongue is Spanish compared to their peers of compulsory secondary education. To test this, a sample of 247 students from Salamanca was selected. Of these 141 students were immigrants. The results show that immigrant students who speak Spanish have more difficulties inferring the meaning of the vocabulary and the expressions that appear in their teaching resources. This leads us to think about the educational practices that we are developing in order to respond the needs of those students.

Key words: immigrant students; cultural social and linguistic diversity; intercultural education; school failure.

\section{SOMMAIRE}

La part d'immigration de l'effectif scolaire dans le système éducatif espagnol est un des facteurs clés expliquant des indices élevés de l'échec scolaire. Face à cette situation, nous prenons comme supposition qu'une partie des difficultés que ces élèves peuvent présenter se justifie du fait de leur méconnaissance des codes culturelles et des moyens de communication qui déterminent le fonctionnement des écoles, lesquelles rendent plus difficiles leur accès aux contenus didactiques, leur communication, leur participation ainsi que leur inclusion éducative. Notre travail a pour but d'identifier les similitudes et les différences dans la compréhension des composantes sociolinguistiques et socioculturelles des enfants provenant des pays hispanophones vis-à-vis de leurs camarades autochtones de «Educación Secundaria 
ELENA MARTÍN-PASTOR, CRISTINA JENARO RÍO Y FRANCISCA GONZÁLEZ-GIL

COMPONENTES SOCIOLINGÜÍSTICOS Y CULTURALES EN EL PROCESO EDUCATIVO DEL ALUMNADO INMIGRANTE

Obligatoria» (Régime d'éducation éspagnol équivalent aux années de collègue et à la première année de lycée). Pour répondre à cet objectif, 247 élèves de Salamanca, dont 141 immigrants, ont participé à une étude. Les résultats dévoilent que les étudiants étrangers hispanophones rencontrent plus de difficultés pour intégrer la signification de certains mots ou expressions employées par les supports didactiques. Ces résultats nous amènent à nous questionner de nouveau sur les pratiques éducatives qui se mettent en place aujourd'hui pour répondre aux besoins de cette catégorie d'élèves.

Mots clés: effectif scolaire immigrant; diversité sociolinguistique et culturelle; éducation interculturelle; échec scolaire.

\section{INTRODUCCIÓN}

En España, el aumento de la presencia de alumnos inmigrantes en las escuelas ha convertido a las aulas en contextos pluriculturales, que generan nuevos desafíos a la práctica docente y a la organización y funcionamiento de los centros, con el fin de ofrecer una respuesta educativa que se adapte a las necesidades de estos escolares. El trabajo que aquí presentamos ha centrado el foco de interés en este colectivo, al constituirse como grupo de riesgo por presentar altos índices de fracaso y abandono escolar. De hecho, un aspecto importante a resaltar es que, a pesar de la existencia de planes y medidas educativas para superar las dificultades con las que el alumnado extranjero se encuentra, y a la luz de los resultados obtenidos por la mayoría de estos estudiantes (Instituto de Evaluación, 2010a, 2010b; OCDE, 2013), debemos cuestionarnos la eficacia de las mismas.

En este sentido, tenemos que destacar que gran parte de las medidas planteadas se centran en la enseñanza del castellano, considerando el desconocimiento del idioma la principal causa de las dificultades académicas de estos escolares, cuando, según datos del MEC, cerca de un 40\% de los estudiantes inmigrantes son hispanoparlantes. Esto nos ha llevado a reflexionar sobre el origen de esas dificultades y profundizar en el conocimiento de las necesidades que presentan estos alumnos, principalmente en aquellas relacionadas con el ámbito comunicativo. Para ello, partimos de la teoría de que el uso que se hace de una determinada lengua y su comprensión viene determinado por el contexto en el que se utiliza y, por tanto, por la cultura en que la sustenta. Ello puede generar un desfase cultural en los escolares que proceden de otros contextos, no sólo lingüísticos, sino también geográficos, que repercuta en la comprensión de textos escolares y, por tanto, en su rendimiento académico.

\section{ESTADO DE LA CUESTIÓN}

Como ya se ha resaltado, el incremento de alumnos extranjeros en el sistema educativo nacional como consecuencia de los fuertes movimientos migratorios de 
sus familias en busca de mejores oportunidades está transformando las aulas en contextos pluriculturales, donde el respeto y la valoración de las diferencias se convierten en pilares fundamentales que deben guiar la educación que se proporciona a todos los estudiantes (Parrilla, 2007). Aun así, Alegre (2008) afirma que la incorporación del alumnado inmigrante a los centros educativos es considerada como un problema de primer orden (debido a los desafíos que esta situación conlleva), que se manifiesta en algunas ocasiones en un bajo rendimiento y en altos índices de fracaso y abandono escolar. De hecho, Stanat y Christensen (2006) señalan que muchos de los estudiantes de nacionalidad extranjera corren el riesgo de no adquirir los conocimientos básicos que les permitirán desenvolverse en la vida diaria. Distintos autores (Essomba, 2014; Etxeberría y Elosegui, 2010; García, Rubio y Bouachra, 2008, 2015; Guzmán, Feliciano y Jiménez, 2008; Huguet y Navarro, 2003; Huguet, Navarro y Janés, 2007; Prado, 2011) justifican esta situación a través de varios factores que pueden incidir en que los estudiantes inmigrantes obtengan peores resultados que sus compañeros: la edad de llegada e inicio de la escolarización, la lengua materna versus la lengua vehicular de la escuela, la trayectoria educativa, las características del entorno social y escolar de acogida, las actitudes y la formación del profesorado, la desigual distribución de estos alumnos en centros públicos y privados, etcétera.

Lo anterior ha supuesto que el escolar inmigrante haya pasado a engrosar la lista de aquellos a los que les acompaña la etiqueta de alumnado con necesidad específica de apoyo educativo y, por tanto, de los que reciben una educación diferenciada a la del resto de sus compañeros, así lo demuestran los diferentes planes de atención a la diversidad en general y a la población inmigrante en particular. Medidas que, escudadas en el apelativo de educación intercultural, poseen un fuerte carácter segregador y compensatorio donde se siguen apreciando las resistencias a la aceptación de la heterogeneidad (lingüística, cultural y de identidad) (García et al., 2008; Ortega, 2013), resultando ineficaces hasta el momento ya que a final de curso cada vez es más común encontrarse unido el fenómeno fracaso escolar con la categoría estudiante extranjero.

De esta forma, un indicador importante a la hora de caracterizar la escolarización de los alumnos inmigrantes es el relativo al bajo rendimiento académico que obtienen estos estudiantes. López y García (2014) resaltan al respecto la fuerte relación existente entre ser de nacionalidad extranjera y tener posibilidades para repetir los cuatro cursos de ESO. Así mismo, los últimos informes PISA advierten que este colectivo obtiene resultados significativamente más bajos que sus compañeros en las principales materias escolares (Instituto de Evaluación, 2010a, 2010b; OCDE, 2013). Así, en diferentes análisis realizados sobre dichos informes (Choi y Calero, 2013; Cordero, Crespo y Pedraja, 2009; Stanat y Christensen, 2006) se recoge la notable incidencia negativa de la condición inmigrante que se asocia al fracaso escolar del alumnado. De hecho, el efecto inmigración, en el periodo 2009-2012, ha penalizado negativamente casi dos puntos en las puntuaciones obtenidas por España en el programa PISA 2012 (OCDE, 2013). 
Todo ello pone de manifiesto que el alumnado extranjero continúa siendo un colectivo de exclusión (al que se le atribuye la categoría de alumno de compensatoria) debido, entre otras cosas, a su desconocimiento de los códigos culturales que determinan el funcionamiento de la escuela, con especial hincapié en lo que se refiere a la lengua vehicular. En este sentido, coincidimos con García et al. (2008, 47) cuando afirman que "la lengua se ha convertido en el argumento profesional y político para desplegar todo tipo de medidas de atención a estos nuevos escolares». Así lo demuestra también el hecho de que los principales mecanismos para actuar con el estudiante inmigrante se concretan en programas de inmersión lingüística y en aulas de acogida (García et al., 2008, 2015; Simó, Pamies, Collet-Sabé y Tort, 2014).

En las distintas provincias de Castilla y León, comunidad donde se ubica el presente estudio, nos encontramos con las Aulas de Adaptación Lingüística y Social (de ahora en adelante, aulas ALISO), diseñadas para proporcionar una respuesta especializada a los alumnos que desconocen la lengua y cultura españolas, y que se ubican en aquellos centros educativos que presentan un mayor índice de alumnado extranjero con un nivel de castellanización nulo o muy bajo. Sin embargo, nos cuestionamos la eficacia de dichas aulas para responder a las necesidades y dificultades de la totalidad de la población inmigrante presente en esta comunidad. Así, al analizar las cifras relativas al curso 2013-2014, proporcionadas por el Ministerio de Educación, nos encontramos con que el 35\% del estudiante extranjero escolarizado en Castilla y León procede de países de América Central y América del Sur, donde, exceptuando Brasil, la lengua materna es el castellano. Sin embargo, son alumnos que igualmente presentan dificultades escolares (Alegre, Benito y González, 2012). Esto nos sitúa en la misma dirección que a López y García (2014) cuando afirman que el llamado "problema", entonces, no se ubica tanto en la condición de extranjero como en un conjunto de condiciones socioculturales que tendemos a resumir en este agrupamiento.

Concretamente, en el presente trabajo, nos centramos en la diversidad lingüística como factor que viene motivado por tres tipos de razones, fuertemente vinculadas a dichas condiciones socioculturales (Prado, 2011): 1) geográficas, que se refiere tanto a las distintas lenguas y dialectos correspondientes a los diferentes lugares de procedencia de las personas, como a las diversas maneras de hablar un mismo idioma en varias zonas geográficas; 2) sociales, en cuanto al uso que se hace de la lengua según la edad, el sexo, la clase sociocultural; y 3) pragmáticas, referidas a la manera de utilizar el lenguaje en función del contexto comunicativo en el que se desarrolle.

Desde esta perspectiva, ponemos nuestro foco en el papel que juega la competencia sociolingüística en el aprendizaje del escolar procedente de países de habla hispana, entendiendo por dicha competencia el conjunto de conocimientos y habilidades necesarios para utilizar un idioma de acuerdo a las exigencias contextuales a nivel social y cultural de un determinado país o zona geográfica (Moreno, 2007). 
Partimos, para ello, del supuesto de que los mediadores comunicacionales de la cultura de un país producen una desigualdad en los estudiantes de otras nacionalidades, generándoles un desfase cultural que dificulta su acceso a los contenidos didácticos, su comunicación, sus relaciones, su participación y, por tanto, su inclusión educativa y social. En esta línea, Salvador Mata y Gutiérrez (2005) señalan que no debemos pasar por alto la relación entre el dominio de una lengua y los factores socioculturales en los que se desarrolla, ya que es un índice del nivel cultural y social del sujeto. Del mismo modo lo reflejan Alegre et al. (2012) al señalar que la lengua se convierte en una marca simbólica distintiva y generadora de categorizaciones y prejuicios. A esto hay que añadir, como ya adelantábamos, que cada grupo humano desarrolla sus propias prácticas discursivas (Cassany, 2006), es decir, una misma expresión cambia de significado en cada lugar y hay algunas palabras que adquieren connotaciones particulares según el contexto en el que se utilizan y el valor que le demos cada uno. Por ello, debemos tener en cuenta que el conocimiento de las palabras y expresiones va a ser crucial para la comprensión de los contenidos didácticos (Alanis, 2004). Esta situación revela la cotidianeidad que experimentan los estudiantes extranjeros procedentes de países de habla hispana que, a pesar de compartir la misma lengua que sus compañeros de nacionalidad española, utilizan códigos comunicativos diferentes. Lo que revela, a su vez, la influencia de la diversidad lingüística y la consecuente importancia del conocimiento y manejo de los componentes sociolingüísticos en sus trayectorias educativas en otro país diferente al propio, independientemente de que comparta un mismo idioma.

A nivel internacional, la creación en el año 2001 del Marco común para las lenguas: aprendizaje, enseñanza y evaluación (MCER), constituye la base para dar respuesta a la variedad lingüística resultante del incremento de la inmigración en Europa y defiende el plurilingüismo desde la necesidad de desarrollar en los alumnos la habilidad de manifestarse en diferentes lenguas o variedades de una misma lengua (Beacco et al., 2010; Llorián, 2011). Además, describe lo que los estudiantes deben aprender con el fin de utilizar un idioma para comunicarse y los conocimientos y destrezas que tienen que desarrollar para poder actuar de manera eficaz, destacando la competencia comunicativa como una de las principales, que a su vez engloba los componentes lingüístico (destrezas léxicas, fonológicas y sintácticas), pragmático (uso funcional de los recursos lingüísticos) y sociolingüístico (condiciones socioculturales del uso de la lengua). Destrezas todas ellas que dependen, entre otras cosas, de las características culturales de la comunidad donde la persona se socializa y aprende (Instituto Cervantes, 2002). Ante este hecho, López y García (2014) o Simó et al. (2014), en otros, destacan la influencia del trabajo que se realiza en los centros escolares, como uno de los principales agentes educativos de dicha comunidad, en los resultados académicos, que, en ocasiones, no hace más que reforzar determinadas posiciones sociales y jerarquizarlas.

La educación intercultural, en relación con lo expuesto, se ha convertido en la respuesta idónea ante la pluralidad cultural y lingüística que caracteriza el actual sistema educativo, y en donde esa diversidad se convierte en un elemento 
enriquecedor para el aprendizaje, a partir del principio de equidad más que del de igualdad (Peñalva y Soriano, 2010). Sin embargo, coincidimos con Rodríguez (2010) en que las prácticas educativas vigentes responden más a un modelo de escuela homogeneizador, donde, como ya comentábamos, se centra la respuesta educativa proporcionada al alumnado inmigrante en la enseñanza del castellano, olvidándose, en cierto modo, de aquellos hispanoparlantes, y excluyendo del currículum y de las actividades escolares la cultura y las experiencias de este colectivo (Alanis, 2004).

Este hecho repercute en la adquisición de aprendizajes de los alumnos procedentes de otros países, aunque éstos sean de habla hispana, ya que los niños de cualquier cultura adquieren los modelos de esa cultura, incluyendo las maneras de comunicarse (Olson, 1997). Del mismo modo lo expresan Salvador Mata y Gutiérrez (2005) al afirmar que la pertenencia a una clase social o a una cultura condiciona, positiva o negativamente, el dominio de la expresión y de la comprensión. Lo anterior da lugar a que lengua y cultura sean conceptos indisociables (Prado, 2011).

En este contexto, las investigaciones que se han llevado a cabo con alumnos inmigrantes y sus dificultades en el ámbito lingüístico y comunicativo se han centrado en el análisis de su proceso de adquisición y conocimiento de la lengua vehicular de la escuela (Huguet, Chireac, Navarro y Sansó, 2011; Huguet y Navarro, 2003; Navarro y Huguet, 2006). Sin embargo, estos estudios no contemplan el componente sociolingüístico y sociocultural que tiñe al idioma de un país de sus propias peculiaridades discursivas (Prado, 2011) y que puede generar a estos escolares problemas para comprender la intención del emisor al manejar unas expresiones, un vocabulario y una estructura comunicativa que difieren de los propios, lo que desemboca en una limitación o en una barrera para su aprendizaje como consecuencia de no tener el alcance lingüístico que la sociedad de acogida les exige.

Así pues, a la vista de lo previamente expuesto, con el presente trabajo pretendemos contribuir a ampliar el estado de los conocimientos existentes respecto a la situación de aquellos alumnos inmigrantes cuya lengua materna es el castellano, y más concretamente en cuanto al dominio de los componentes sociolingüísticos y socioculturales, con el fin de aportar una perspectiva diferente del tema que complemente a lo ya existente. Concretamente, nos planteamos identificar semejanzas y diferencias en la comprensión de componentes sociolingüísticos y socioculturales de niños procedentes de países de habla hispana, frente a sus iguales españoles. Hipotetizamos además que: (1) existirán diferencias en la comprensión de los aspectos sociolingüísticos y socioculturales; (2) los años de residencia en España influirán de manera positiva en el dominio de dichos aspectos.

\section{MÉTODO}

Se trata de un estudio descriptivo-correlacional y con medidas ex post facto, en el que hemos empleado análisis descriptivos e inferenciales multivariados y bivariados. 


\subsection{Participantes}

Los participantes consistieron en una muestra de conveniencia, que aceptó voluntariamente participar, tras garantizárseles el anonimato. Dicha muestra estuvo formada por 247 alumnos que cursaban la Educación Secundaria Obligatoria (un $28.3 \%$ primero, un $31.6 \%$ segundo, un $19.8 \%$ tercero y un $20.2 \%$ cuarto) en siete centros educativos (cinco públicos) de la provincia de Salamanca (España). Éstos se dividían en un $54,7 \%$ de alumnado inmigrante procedente de países latinoamericanos y de habla hispana, mayoritariamente de Bolivia $(27,4 \%)$, Colombia $(25,9 \%)$, Ecuador y Perú (cada uno con un 13,32\%), y con un tiempo de residencia en España inferior a 6 años (67,4\%); y un 45.3\% de estudiantes de nacionalidad española. En cuanto al género de los participantes, un $55.5 \%$ eran mujeres.

Con la finalidad de comprobar la igualdad en las distribuciones poblacionales de cada uno de los subgrupos en los que se dividía la muestra (inmigrantesespañoles), realizamos contrastes de homogeneidad mediante Chi Cuadrado en cuanto a género, nivel educativo (determinado por el curso en el que estaba escolarizada la muestra) y nivel sociocultural del padre y de la madre; considerados éstos algunos de los factores que más repercuten en el rendimiento educativo del alumnado (Calero, Choi y Waisgrais, 2010; Choi y Calero, 2013; Cordero et al., 2013; Lorenzo, Santos Rego y Godás, 2012; OCDE, 2013) y que, por tanto, más podían influir en los resultados obtenidos por los participantes de este estudio. Los análisis pusieron de manifiesto la ausencia de diferencias en función del género $\left(\chi^{2}=1.735 ; \mathrm{gl}=1 ; \mathrm{p}=.188\right)$, nivel educativo $\left(\chi^{2}=6.877 ; \mathrm{gl}=3 ; \mathrm{p}=.076\right)$, nivel sociocultural del padre $\left(\chi^{2}=9.190 ; \mathrm{gl}=4 ; \mathrm{p}=.057\right)$, y nivel sociocultural de la madre $\left(\chi^{2}=4.267 ; \mathrm{gl}=4 ; \mathrm{p}=.371\right)$.

\subsection{Instrumento}

La recogida de datos se llevó a cabo a través de una prueba ad hoc con la que se preguntaba a los alumnos sobre el significado del vocabulario y algunas expresiones recogidas en un texto que era diferente para cada nivel educativo que se extrajo de los libros de Lengua Castellana y Literatura con los que habitualmente se trabaja en las aulas, con el fin de que dichos textos se adaptasen al nivel educativo de los alumnos y, por tanto, a lo establecido en el currículo. En cada caso, el alumno tenía que, por un lado, indicar las palabras que le resultaban desconocidas, de las que posteriormente se realizaba un recuento y se categorizaban en sustantivos, adjetivos y verbos (con objeto de valorar el tipo de palabra menos conocida por el alumno). Por otro lado, el escolar debía explicar el significado de cuatro expresiones coloquiales, seleccionadas al azar, en función del sentido que adquirían dentro de cada texto; el significado que cada participante otorgó a cada una de las expresiones fue puntuado por consenso entre un grupo de jueces expertos a partir de criterios previamente establecidos. De este modo, las respuestas fueron 
ELENA MARTÍN-PASTOR, CRISTINA JENARO RÍO Y FRANCISCA GONZÁLEZ-GIL

baremadas en una escala de 1 a 4 donde cada puntuación se correspondía con las siguientes afirmaciones ${ }^{2}$ :

$1=$ No transmite el significado de la frase o no aporta ninguna respuesta .

$2=$ Identifica parte del significado de la frase por alguna palabra incluida en la misma.

$3=$ Identifica parcialmente el significado de la frase.

$4=$ Describe correctamente lo que quiere decir la frase en el contexto en el que se está utilizando dentro del texto.

\subsection{Procedimiento}

Para conocer los datos de población inmigrante que cursaba ESO en Salamanca, contactamos con la Dirección Provincial de Educación, que nos proporcionó los porcentajes de alumnos extranjeros, por centros, en esta etapa educativa. Solicitamos su autorización para la realización del presente estudio y nos pusieron en contacto con los profesores encargados de las aulas ALISO, con los que concertamos una entrevista. En ella nos informaron de que su trabajo se centraba fundamentalmente en aquellos estudiantes que no sabían castellano (enseñanza de gramática y vocabulario básico, facilitando el acceso a las áreas curriculares), por lo que no podían participar en nuestro trabajo. Por ello, nos remitieron al equipo directivo de cada centro educativo.

La información recopilada nos permitió seleccionar aquellos centros que contaban en sus aulas con un mayor número de alumnos inmigrantes de origen latino. Se estableció contacto con los mismos, por correo electrónico y vía telefónica, y posteriormente se mantuvieron entrevistas con el director, orientador y jefe de estudios de cada centro para resolver las posibles dudas. Conseguidas las autorizaciones, se procedió a organizar y poner en marcha la evaluación en cada centro.

\section{RESUltados}

Con el fin de conocer el dominio de vocabulario de los participantes, en primer lugar se realizó un recuento del número de palabras desconocidas por cada uno de ellos. En la Tabla 1 se puede observar que los alumnos inmigrantes, pese a que su lengua materna sea el español, desconocen el significado de un mayor número de palabras que el alumnado autóctono, aspecto que se traduce en una media más elevada (1.01) (rango de 0 a 6 palabras desconocidas para este grupo, frente a un rango de 0 a 4 para el grupo de los españoles), resultando dicha diferencia significativa $(\mathrm{p}<.05)$.

2. En el apartado correspondiente a resultados se mostrarán algunos ejemplos de las respuestas proporcionadas por los participantes y su correspondiente puntuación. 
TABLA 1

DiFERENCIAS EN MANEJO DE VOCABULARIO (MUESTRA TOTAL)

\begin{tabular}{|c|c|c|c|c|c|}
\hline & $\mathrm{N}$ & MEDIA & DESVIACIÓN TÍPICA & $\mathrm{T}$ & SIG. \\
\hline $\begin{array}{c}\text { Inmigrantes } \\
\text { hispanohablantes }\end{array}$ & 135 & 1.01 & 1.40 & 1.993 & .048 \\
\hline Españoles & 112 & 0.71 & .81 & & \\
\hline
\end{tabular}

También se analizó la posible asociación entre la procedencia de los alumnos (España vs. Países latinos hispanohablantes) y la categoría de vocabulario con la que los participantes presentaban más dificultades (sustantivo, adjetivo, verbo y combinaciones de esos tres tipos de palabras). Los análisis evidenciaron una asociación significativa entre ambas variables (procedencia y tipo de vocabulario) (Chi cuadrado $=19.407 ; \mathrm{gl}=3 ; \mathrm{p}=.00)$. Además, el análisis de los residuos tipificados corregidos (Haberman, 1973) indicó que el alumnado inmigrante desconoce un mayor número de combinaciones de tipos de palabras, mientras que conoce más adjetivos que sus compañeros españoles (Tabla 2).

TABLA 2

FRECUENCIA Y PORCENTAJE DE TIPOS DE PALABRAS DESCONOCIDAS POR LOS PARTICIPANTES

\begin{tabular}{|l|c|c|c|c|}
\hline \multicolumn{1}{|c|}{ GRUPO } & SUSTANTIVO & ADJETIVO & VERBO & $\begin{array}{c}\text { COMBINACIÓN DE } \\
\text { TIPOS DE PALABRAS }\end{array}$ \\
\hline Inmigrante latino & $28(38.9 \%)$ & $14(19.4 \%)$ & $4(5.6 \%)$ & $26(36.1 \%)$ \\
\hline Residuos tipificados & 1.2 & -4.2 & .7 & 3.0 \\
\hline Español & $17(27.9 \%)$ & $34(55.7 \%)$ & $2(3.3 \%)$ & $8(13.1 \%)$ \\
\hline Residuos tipificados & -1.2 & 4.2 & -.7 & -3.0 \\
\hline
\end{tabular}

En lo que respecta a la comprensión de las expresiones coloquiales, como comentábamos con anterioridad, las respuestas fueron baremadas en una escala de 1 a 4 por consenso entre jueces expertos según los criterios previamente establecidos. En la Tabla 3 presentamos ejemplos del significado que algunos alumnos otorgaron a las expresiones coloquiales incluidas en los textos que manejaron; concretamente, en cada columna se muestra una de las expresiones seleccionadas por cada curso educativo en el que estaban matriculados los alumnos, mientras que cada fila refleja la puntuación obtenida. 
ELENA MARTÍN-PASTOR, CRISTINA JENARO RÍO Y FRANCISCA GONZÁLEZ-GIL

TABLA 3

RESPUESTAS PROPORCIONADAS POR ALUMNOS RESPECTO AL SIGNIFICADO DE EXPRESIONES COLOQUIALES Y PUNTUACIÓN

\begin{tabular}{|c|c|c|c|c|}
\hline & $\begin{array}{c}1 .^{\circ} \text { ESO } \\
\text { "DECIR ALGO } \\
\text { QUE ROMPA } \\
\text { EL HIELO» }\end{array}$ & $\begin{array}{c}2 .{ }^{\circ} \text { ESO } \\
\text { "DIME CÓMO } \\
\text { HABLAS Y TE } \\
\text { DIRÉ QUIÉN ERES» }\end{array}$ & $\begin{array}{c}3 .^{\circ} \text { ESO } \\
\text { «ESA CARRERA } \\
\text { QUE PUEDE SER } \\
\text { EL NO VA MÁS» }\end{array}$ & $\begin{array}{l}4^{\circ}{ }^{\circ} \text { ESO } \\
\text { «LA FE MUEVE } \\
\text { MONTAÑAS» }\end{array}$ \\
\hline $\begin{array}{l}\text { (1) No transmite el } \\
\text { significado de la } \\
\text { frase o no aporta } \\
\text { ninguna respuesta }\end{array}$ & No sé & $\begin{array}{c}\text { Saber si sabes } \\
\text { hablar o no }\end{array}$ & $\begin{array}{l}\text { Si no gana, } \\
\text { ya no podrá } \\
\text { ganar jamás }\end{array}$ & $\begin{array}{l}\text { Tienes que } \\
\text { creer en algo }\end{array}$ \\
\hline $\begin{array}{l}\text { (2) Identifica parte } \\
\text { del significado de } \\
\text { la frase por alguna } \\
\text { palabra incluida } \\
\text { en la misma }\end{array}$ & Hablar & $\begin{array}{c}\text { Cuando una } \\
\text { persona babla } \\
\text { distinto, sabes } \\
\text { que es de } \\
\text { otro país }\end{array}$ & $\begin{array}{c}\text { Va a ser } \\
\text { importante }\end{array}$ & $\begin{array}{l}\text { Necesitas fe } \\
\text { para hacer } \\
\text { cosas }\end{array}$ \\
\hline $\begin{array}{l}\text { (3) Identifica } \\
\text { parcialmente } \\
\text { el significado } \\
\text { de la frase }\end{array}$ & $\begin{array}{l}\text { Lanzarse } \\
\text { a hablar }\end{array}$ & $\begin{array}{l}\text { De la forma en } \\
\text { que te expreses } \\
\text { sabré si eres } \\
\text { educado o no }\end{array}$ & $\begin{array}{l}\text { Puede ser la } \\
\text { mejor de todas }\end{array}$ & $\begin{array}{c}\text { Con la fe } \\
\text { puedes, te } \\
\text { atreves a hacer } \\
\text { cosas dificiles }\end{array}$ \\
\hline $\begin{array}{l}\text { (4) Describe } \\
\text { correctamente lo } \\
\text { que quiere decir } \\
\text { la frase en el } \\
\text { contexto en el que } \\
\text { se está utilizando } \\
\text { dentro del texto }\end{array}$ & $\begin{array}{c}\text { Comenzar a } \\
\text { bablar sobre } \\
\text { cualquier tema } \\
\text { sin importancia } \\
\text { para } \\
\text { comenzar una } \\
\text { conversación } \\
\text { y no estar } \\
\text { incómodos con } \\
\text { el silencio }\end{array}$ & $\begin{array}{l}\text { La manera de } \\
\text { bablar define } \\
\text { a una persona }\end{array}$ & $\begin{array}{l}\text { La carrera más } \\
\text { emocionante } \\
\text { e importante } \\
\text { del Mundial } \\
\text { que nos puede } \\
\text { traer grandes } \\
\text { sorpresas }\end{array}$ & $\begin{array}{l}\text { Si tienes fe } \\
\text { puedes hacer } \\
\text { y conseguir } \\
\text { todo lo que te } \\
\text { propongas }\end{array}$ \\
\hline
\end{tabular}

Una vez recogidas las puntuaciones obtenidas por cada alumno procedimos a realizar análisis multivariados para determinar si, tomadas todas las variables conjuntamente, la procedencia de los participantes (España vs. Países latinos hispanohablantes) afectaba significativamente a los resultados. De este modo, si el estadístico de contraste (Lambda de Wilks) era significativo, procedíamos a realizar los contrastes bivariados para identificar en qué variables existían diferencias significativas. El análisis puso de manifiesto la existencia de estas diferencias en el conocimiento de expresiones coloquiales entre los alumnos inmigrantes de habla hispana y el alumnado español (Lambda de Wilks = .512; $\mathrm{gl}=4.249 ; \mathrm{F}=59.331$; $\mathrm{p}=.00)$. En segundo lugar, la realización de contrastes bivariados reflejó la existencia de diferencias significativas en todas las expresiones analizadas. La Tabla 4 
permite apreciar cómo los alumnos inmigrantes muestran mayores dificultades para reconocer el significado de las expresiones y frases hechas seleccionadas.

TABLA 4

COMPARACIÓN DE MEDIAS Y SIGNIFICACIÓN (PRUEBA T DE STUDENT) EN EXPRESIONES COLOQUIALES DE LOS PARTICIPANTES

\begin{tabular}{|c|c|c|c|c|c|}
\hline ÍTEM & GRUPO & MEDIA & DESV. TÍP. & $\mathrm{N}$ & T \\
\hline Expresión 1 & & & & & $-7.530^{*}$ \\
\hline & Inmigrantes & 2.25 & 1.268 & 135 & \\
\hline & Españoles & 3.36 & .985 & 112 & \\
\hline Expresión 2 & & & & & $-9.326^{*}$ \\
\hline & Inmigrantes & 1.97 & 1.139 & 135 & \\
\hline & Españoles & 3.28 & 1.042 & 112 & \\
\hline Expresión 3 & & & & & $-7.319^{*}$ \\
\hline & Inmigrantes & 1.95 & 1.230 & 135 & \\
\hline & Españoles & 3.08 & 1.187 & 112 & \\
\hline Expresión 4 & & & & & $-10.751^{*}$ \\
\hline & Inmigrantes & 2.01 & 1.123 & 135 & \\
\hline & Españoles & 3.45 & .948 & 112 & \\
\hline
\end{tabular}

*SIGNIF. Con $\mathrm{p}<.005$

Además, se analizó la influencia que ejercían los años de residencia en España en la mejora de la comprensión de expresiones coloquiales y en el dominio del vocabulario. Para ello, en primer lugar se recodificó la variable "años en España" en tres subgrupos: $1 .^{\circ}$ de 1 a 3 años, 2. ${ }^{\circ}$ de 4 a 6 años y $3 .^{\circ}$ más de 6 años; para, posteriormente, realizar un contraste de medias (análisis de varianza). Como se muestra en la Tabla 5, al contrario de lo que hipotetizábamos, no existen diferencias significativas $(\mathrm{p}>.05)$ en el manejo de vocabulario, ni en el conocimiento de las expresiones coloquiales incluidas en sus libros de texto en función del tiempo residido en el país de acogida. 
ELENA MARTÍN-PASTOR, CRISTINA JENARO RÍO Y FRANCISCA GONZÁLEZ-GIL

COMPONENTES SOCIOLINGÜISTICOS Y CULTURALES EN EL PROCESO EDUCATIVO DEL ALUMNADO INMIGRANTE

TABLA 5

ESTADÍSTICOS DESCRIPTIVOS Y DE SIGNIFICACIÓN DE LAS DIFERENCIAS (ANOVA) EN LA COMPRENSIÓN DE VOCABULARIO Y EXPRESIONES EN FUNCIÓN DE LOS AÑOS DE RESIDENCIA EN ESPAÑA

\begin{tabular}{|c|c|c|c|c|c|c|}
\hline ÍTEM & GRUPO & MEDIA & DESV. TÍP. & $\mathrm{N}$ & $\mathrm{F}$ & SIG. \\
\hline \multirow[t]{4}{*}{ Vocabulario desconocido } & & & & & .393 & .676 \\
\hline & De 1 a 3 años & 1.10 & 1.683 & 39 & & \\
\hline & De 4 a 6 años & .87 & 1.372 & 52 & & \\
\hline & Más de 6 años & 1.07 & 1.163 & 44 & & \\
\hline \multirow[t]{4}{*}{ Expresión 1} & & & & & .266 & .767 \\
\hline & De 1 a 3 años & 2.15 & 1.268 & 39 & & \\
\hline & De 4 a 6 años & 2.35 & 1.235 & 52 & & \\
\hline & Más de 6 años & 2.23 & 1.327 & 44 & & \\
\hline \multirow[t]{4}{*}{ Expresión 2} & & & & & 1.024 & .362 \\
\hline & De 1 a 3 años & 1.95 & 1.191 & 39 & & \\
\hline & De 4 a 6 años & 1.83 & 1.098 & 52 & & \\
\hline & Más de 6 años & 2.16 & 1.140 & 44 & & \\
\hline \multirow[t]{4}{*}{ Expresión 3} & & & & & 1.187 & .308 \\
\hline & De 1 a 3 años & 1.82 & 1.189 & 39 & & \\
\hline & De 4 a 6 años & 2.15 & 1.334 & 52 & & \\
\hline & Más de 6 años & 1.82 & 1.126 & 44 & & \\
\hline \multirow[t]{4}{*}{ Expresión 4} & & & & & .395 & .674 \\
\hline & De 1 a 3 años & 1.92 & 1.222 & 39 & & \\
\hline & De 4 a 6 años & 2.12 & 1.166 & 52 & & \\
\hline & Más de 6 años & 1.95 & .987 & 44 & & \\
\hline
\end{tabular}

\section{CONCLUSIONES Y DISCUSIÓN}

Al inicio de este artículo señalábamos como, en el ámbito educativo, las principales intervenciones llevadas a cabo con el alumnado inmigrante han ido encaminadas a la enseñanza de la lengua vehicular de la escuela. De hecho, la mayoría de las autonomías disponen de aulas de acogida en sus centros educativos (como, por ejemplo, las aulas ALISo en Castilla y León), al tiempo que se han desarrollado proyectos en el contexto escolar como "Lenguas de escolarización» o el Plan Curricular del Instituto Cervantes. Medidas todas ellas centradas en la enseñanza del castellano y dirigidas a sólo un sector de la población escolar extranjera, aquella que desconoce la lengua vehicular de la escuela. Sin embargo, al analizar 
la realidad educativa, nos encontramos con que más de un cuarto del alumnado inmigrado proviene de países de habla hispana y, a pesar de que, aparentemente, la barrera idiomática está superada, son estudiantes que también presentan bajos índices en su rendimiento escolar (García et al., 2008, 2015; Guzmán et al., 2008). Lo anterior nos llevó a cuestionarnos si realmente el manejo de la lengua resultaba suficiente y a responder, coincidiendo con García et al. (2015), que no, ya que, a pesar de que los países de origen del alumnado inmigrante compartan el idioma con el país de acogida, nos encontramos ante realidades socioculturales diferentes que tienen su reflejo en la manera de utilizar dicho idioma (Carrasco y Gibson, 2010; Moreno, 2007; Olson, 1997; Prado, 2011), influyendo también en el aprendizaje del alumnado.

En este sentido, al analizar los resultados obtenidos podemos concluir que los alumnos procedentes de países latinos de habla hispana poseen un mayor desconocimiento del vocabulario incluido en los textos de sus libros de Lengua Castellana y Literatura, aspecto fundamental que de entrada les dificultaba el acceso a los contenidos didácticos que se imparten en el aula, ya que, como señala Alanis (2004), las palabras son herramientas que utilizamos para acceder al conocimiento, expresar ideas y aprender nuevos conceptos, lo que hace que el manejo del vocabulario por los alumnos esté estrechamente ligado a su éxito escolar. También Salvador Mata, Gallego y Mieres (2007) inciden en que el dominio léxico favorece la comprensión lectora y la adquisición de nuevos aprendizajes. A ello debemos añadir los problemas que presentaron para inferir el significado de las expresiones coloquiales seleccionadas y que formaban parte también de los textos con los que habían trabajado. Los alumnos inmigrantes, en comparación con sus compañeros autóctonos, presentaban más dificultades para identificar el significado de dichas expresiones o, únicamente a partir del conocimiento de una de las palabras incluidas en la frase, identificaban una ligera parte del mismo (véase Tabla 3). Ante esta situación, desconocimiento del significado de un mayor número de palabras y de las expresiones coloquiales que formaban parte de los libros de texto, estos alumnos ya cuentan con una desventaja respecto a sus compañeros, producto de la interacción con un texto escrito en otra cultura diferente a la suya y, por tanto, con unas prácticas discursivas distintas (Cassany, 2006). De hecho, López y García (2015) advierten que las diferencias culturales entre unos lugares y otros influyen en los resultados académicos del alumnado de nacionalidad extranjera, siendo dichos resultados peores que los obtenidos por sus compañeros nativos.

Alegre et al. (2012), en una investigación que analizaba las experiencias de los alumnos inmigrantes en su incorporación al sistema educativo español, resaltaban como revelador el hecho de que muchos de los estudiantes latinoamericanos entrevistados hacían referencia a la diferencia idiomática como variable explicativa en el establecimiento de pobres relaciones con otros compañeros, y como marca simbólica distintiva generadora de categorizaciones que tienen también su repercusión en la adquisición de aprendizajes por parte de estos estudiantes. En la misma línea, García et al. (2008) recogen el análisis realizado por Carabaña (2007) sobre 
distintos informes PISA, en el que concluye que los inmigrantes cuya lengua materna es el castellano aprenden menos que aquellos que poseen otro idioma; advirtiendo que las explicaciones centradas en la lengua y en el tiempo de estancia en el país de acogida para justificar el menor rendimiento del alumnado extranjero en comparación con el autóctono no pueden generalizarse, ya que dependen de cada caso concreto.

En este sentido, si tomamos como referencia la variable "años en España», los resultados obtenidos ponían de manifiesto la ausencia de diferencias significativas entre el alumnado inmigrante hispanoparlante, en función de cuál fuera su tiempo de residencia, en el dominio del vocabulario y expresiones coloquiales con los que los alumnos pueden encontrarse en los libros de texto que manejan en el aula. Al revisar investigaciones que abordan esta variable, observamos que la mayoría la han estudiado como factor a tener en cuenta en el aprendizaje de la lengua vehicular de la escuela por alumnos inmigrantes cuya lengua materna difiere del castellano (Huguet et al., 2011; Huguet et al., 2007; Navarro y Huguet, 2006; Rodríguez, 2010). Aun así algunos resaltan que los estudiantes extranjeros, con independencia de que su idioma de origen sea o no el castellano, no llegan a igualar al alumnado español en el dominio de la lengua, a pesar de que su permanencia en España sea superior a seis años (Huguet et al., 2007).

Todo lo anterior nos lleva a cuestionarnos nuevamente si realmente son suficientes las acciones que las administraciones educativas están llevando a cabo a través de los programas de inmersión lingüística, compensatorios y de enseñanza del español como segunda lengua (L2) y que, al tiempo, constituyen prácticamente la única respuesta a las necesidades de estos alumnos, centrando el aprendizaje de la competencia comunicativa en la adquisición de aspectos gramaticales, y dejando de lado, en la mayoría de las ocasiones, el componente social y cultural que influye en el uso que se haga de una lengua en función del contexto donde ésta se desarrolle (Prado, 2011). En este sentido, resaltamos el papel decisivo que los centros educativos juegan en el diseño de estrategias y acciones educativas que realmente den respuesta a las necesidades del alumnado inmigrante, desarrollando, como plantean Etxeberría y Elosegui (2010), intervenciones que garanticen el progreso académico de estos estudiantes a largo plazo, en lugar de esperar soluciones de arreglo rápido a corto plazo.

Un agente decisivo en este proceso es el profesorado. Así, todas las comunidades han impulsado cursos de formación para este colectivo como herramienta fundamental para el tratamiento del fenómeno migratorio en España (Martínez Lizarrondo, 2009). En el caso de Castilla y León, éstas se han desarrollado desde el Centro de Recursos de Educación Intercultural (CREI) y los Centros de Formación e Innovación Educativa (CFIE). Sin embargo, estas acciones formativas, en muchas ocasiones, no han logrado responder a las necesidades de los docentes al no concretarse en el desarrollo de habilidades y competencias para poner en marcha procesos de transformación de la educación escolar hacia valores interculturales. Además, son recursos, al igual que ocurre con las aulas Aliso, que se han venido 
fomentando prácticamente solo en los centros donde se utilizan medidas de compensación educativa debido a la elevada tasa de alumnos inmigrantes en sus aulas (Martínez Lizarrondo, 2009). Por ello, debemos plantear acciones que abarquen a toda la comunidad educativa y plantearse como una filosofía en torno a la cual se debe reorganizar la educación que proporcionamos a todos los alumnos y el funcionamiento de los centros escolares.

Consideramos, en este sentido, que un primer paso para seguir avanzado y mejorando la respuesta educativa que se proporciona al alumnado, en este caso con especial hincapié en aquel procedente de otros países, aunque éstos sean de habla hispana, es el diseño de un currículo que tenga en cuenta las posibles limitaciones lingüísticas de los estudiantes inmigrantes, así como los factores que determinan el proceso de adquisición o el grado de dominio sociocultural de la lengua vehicular de la escuela (edad, procedencia, rasgos culturales, objetivos educativos, etc.) y familiarizarse con todo el universo cultural que determina el uso de la lengua en un determinado contexto. Coincidimos así con Carrasco (2001) que opina que hay que considerar tres dimensiones de los alumnos para entender el aprendizaje del castellano o cualquier otro idioma. Éstas son la distancia cultural, la jerarquía lingüística según la posición social de cada grupo y las situaciones de uso de las lenguas; y es por esto por lo que su enseñanza debe vincularse a contextos significativos para los escolares. Por lo que de nuevo se pone de manifiesto la necesidad de incluir en el currículum escolar elementos lingüísticos, sociales y culturales relacionados con las experiencias de todos los alumnos inmigrantes que forman parte del sistema educativo (Alanís, 2004; Celedón-Pattichis, 2004).

En este escenario, coincidimos con Prado (2011) al plantear que la escuela debe ser intercultural, en concordancia con las características de la sociedad en la que se ubica, a partir de la consideración de la diversidad como riqueza y valorando el bagaje cultural y lingüístico de todos los alumnos. Y en donde el plurilingüismo sea el enfoque desde el que se desarrollen los procesos de enseñanza-aprendizaje (Beacco et al., 2010) y su filtrado a las prácticas que tienen lugar en las aulas, porque, coincidiendo con Llorián (2011), un currículo que se enmarque en dicho enfoque constituiría una posible solución para la inclusión social, lingüística y cultural del alumnado extranjero.

\section{REFERENCIAS BIBLIOGRÁFICAS}

Alanis, I. (2004) Effective Instruction Integrating Language and Literacy, en SALINAS, C. y FranQuiz, M. C. (eds.) Scholars in the field: the challenges of migrant education. Charleston, ERIC, 211-224.

Alegre, M. Á. (2008) Educación e Inmigración. ¿Un binomio problemático? Revista de Educación, 345, 61-82.

Alegre, M. Á.; Benito, R. y González Motos, S. (2012) Experiencias escolares iniciales del alumnado inmigrado: comienzos que marcan. Educación XX1, 15 (2), 137-158. 
Beacco, J.; Byram, M.; Cavalli, M.; Coste, D.; Cuenat, M. E.; Goullier, F. y Panthier, J. (2010) Guide for the development and implementation of curricula for plurilingual and intercultural education. Estrasburgo, Consejo de Europa. Consultado el 20 de noviembre de 2013. http://www.coe.int/t/dg4/linguistic/Source/Source2010_ForumGeneva/ GuideEPI2010_EN.pdf.

Calero, J.; Choi, Á. y Waisgrais, S. (2010) Determinantes del riesgo de fracaso escolar en España: una aproximación a través de un análisis multinivel aplicado a PISA-2006. Revista de Educación, núm. extraordinario, 225-256.

CARRASCO, S. (2001) Educación, aculturación y género. Reflexiones desde la investigación en el nuevo contexto multicultural de Cataluña. Revista Nómadas, 14, 50-66.

Carrasco, S. y Gibson, M. A. (2010) La educación de jóvenes de origen inmigrante en secundaria: algunas lecciones comparativas desde Cataluña y California. Revista del Ministerio de Trabajo e Inmigración, 90, 59-76.

Cassany, D. (2006) Tras las líneas. Sobre la lectura contemporánea. Barcelona, Anagrama.

Celedón-Pattichis, S. (2004) Alternative Secondary Mathematics Programs for Migrant Students: Cultural and Linguistic Considerations, en Salinas, C. y Franquiz, M. E. (eds.) Scholars in the Field: The Challenges of Migrant Education. Charleston, ERIC

Chor, Á. y Calero, J. (2013) Determinantes del riesgo de fracaso escolar en España en PISA2009 y propuestas de reforma. Revista de Educación, 362, 562-593.

Cordero, J. M.; Crespo, E. y Pedraja, F. (2013) Rendimiento educativo y determinantes según PISA: una revisión de la literatura en España. Revista de Educación, 362, 273-297.

Essomba, M. A. (2014) Políticas de la escolarización del alumnado de origen extranjero en el Estado español hoy. Análisis y propuestas. Revista Electrónica Interuniversitaria de Formación del Profesorado, 17 (2), 13-27.

Etxeberría, F. y Elosegui, K. (2010) Integración del alumnado inmigrante: Obstáculos y propuestas. Revista Española de Educación Comparada, 16, 235-263.

García, F. J.; Rubio, M. y Bouachra, O. (2008) Población inmigrante y escuela en España: un balance de investigación. Revista de Educación, 345, 23-60.

García, F. J.; Rubio, M. y Bouachra, O. (2015) Immigrant students at school in Spain: constructing a subject of study. Dve domovini-Two Homelands, 41, 35-46.

Guzmán, R.; Feliciano, L. A. y Jiménez, A. B. (2008) Dificultades de aprendizaje del alumnado inmigrante hispano: la perspectiva de los coordinadores de Programas de Educación Intercultural. Revista de Educación, 355, 547-570.

Haberman, S. J. (1973) The analysis of residuals in cross-classification tables. Biometrics, 29, 205-220.

Huguet, Á.; Chireac, S. M. a ; NAvarro, J. L. y Sansó, C. (2011) Tiempo de estancia y aprendizajes lingüísticos. El caso de los escolares inmigrantes en Cataluña. Cultura y Educación, 23 (3), 355-370.

Huguet, Á. y Navarro, J. L. (2003) Inmigración y conocimiento de la lengua castellana: el caso de los escolares inmigrados en Aragón. Alazet: Revista de Filología, 15, 343-364.

Huguet, Á.; NAvarro, J. L. y JanÉs, J. (2007) La adquisición del castellano por los escolares inmigrantes. El papel del tiempo de estancia y la lengua familiar. Anuario de Psicología, 38 (3), 357-375.

Instituto Cervantes (2002) Marco común europeo de referencia para las lenguas: aprendizaje, enseñanza, evaluación. Madrid, Ministerio de Educación, Cultura y Deporte, Subdirección General de Cooperación Internacional y Grupo ANAYA. 
InSTITUTO De EvaluACión (2010a) Evaluación general de diagnóstico 2009. Educación Primaria. Cuarto curso. Informe de resultado. Madrid, Ministerio de Educación.

InSTITUTO De EVAluación (2010b) PISA 2009. Programa para la Evaluación Internacional de los Alumnos. OCDE. Informe español. Madrid, Ministerio de Educación.

Llonián, S. (2011) Hacia un sistema de referencia único para el alumnado inmigrante: el papel del Marco común europeo de referencia, en Ballano, I. (coord.) Evaluar la competencia lingüística del alumnado inmigrante. Bilbao, Deusto Digital, Universidad de Deusto, 13-34.

López, C. y GarCía, F. J. (2014) Cuestionando categorías de investigación. Resultados académicos de escolares de nacionalidad extranjera y nacionales en un centro de Educación Secundaría. Revista Internacional de Estudios Migratorios, 4 (1), 1-40.

Lorenzo, M.; SAntos Rego, M. Á. y GodÁs, A. (2012) Inmigración y Educación. ¿Influye el nivel educativo de los padres en el rendimiento académico de los hijos? Teoría de la Educación. Revista Interuniversitaria, 24 (2), 129-148.

Martínez Lizarrondo, A. (2009) Políticas autonómicas de integración de inmigrantes: la educación. Revista Española de Educación Comparada, 15, 251-276.

Moreno, F. (2007) Adquisición de segundas lenguas y sociolingüística. Revista de Educación, $343,55-70$.

Navarro, J. L. y Huguet, Á. (2006) Acerca de la competencia lingüística del alumnado de origen inmigrante en educación secundaria. Revista Interuniversitaria de Formación de Profesorado, 56, 69-80.

OCDE (2013) PISA 2012. Programa para la evaluación internacional de los alumnos. Informe español. Volumen I: Resultados y Contexto. Madrid, Ministerio de Educación, Cultura y Deporte

Olson, D. (1998) El mundo sobre el papel. El impacto de la escritura en la estructura del conocimiento. Barcelona, Gedisa.

Ortega, P. (2013) La pedagogía de la alteridad como paradigma de la educación intercultural. Revista Española de Pedagogía, 256, 401-422.

Parrilla, Á. (2007) Inclusive Education in Spain: a view from inside, en BarTon, L. y Amstrong, F. (eds.) Policy, Experience and Change: Cross Cultural Reflections on Inclusive Education. Dordrecht, Springer, 19-36.

PeÑalva, A. y Soriano, E. (2010) Objetivos y contenidos sobre interculturalidad en la formación inicial de educadores y educadoras. Estudios sobre Educación, 18, 37-57.

PRADO, J. (2011) Didáctica de la lengua y la literatura para educar en el siglo XXI. Madrid, La Muralla.

RodrígueZ, R. M. ${ }^{a}$ (2010) Éxito y fracaso escolar de la primera y segunda generación de estudiantes de origen inmigrante. Estudios sobre Educación, 19, 97-118.

Salvador Mata, F.; Gallego, J. L. y Mieres, C. (2007) Habilidades lingüísticas y comprensión lectora. Una investigación empírica. Bordón, 59 (1), 153-166.

Salvador Mata, F. y Gutiérrez, R. (2005) Atención educativa al alumnado con dificultades en lectura y escritura. Málaga, Aljibe.

Simó, N.; Pamies, J.; Collet-Sabé, J. y Tort, A. (2014) La acogida educativa en los centros escolares en Cataluña: más allá de los recursos específicos para el alumnado de nueva incorporación. Revista Complutense de Educación, 25 (1), 177-194.

STANAT, P. y Christensen, G. (2006) Where immigrant students succeed. A comparative review of performance and engagement in PISA 2003. Paris, OCDE. 